\title{
Estrategia metodológica formativa para una docencia enfocada en el desarrollo de competencias
}

Fundamentos teóricos y pedagógicos

TEREsa Ríos*

Los cambios curriculares en la educación superior chilena en los últimos

Palabras clave

15 años no han estado acompañados de manera armónica por una transformación de la docencia universitaria. Esta discordancia entre currículo y docencia efectivamente realizada resulta un impedimento para obtener los resultados que las innovaciones buscan. Se exponen los fundamentos de una estrategia metodológica formativa para una docencia enfocada en el desarrollo de competencias que permita un cambio de las prácticas de enseñanza, de manera que estas innovaciones curriculares ganen en consistencia y respondan mejor a los desafíos que les dieron origen. Se formula una articulación teórico-práctica que da sostén a un nuevo modo de enseñanza en coherencia con las innovaciones curriculares, y al mismo tiempo, atiende la tensión que se produce en el proceso de formación de un profesional universitario entre la demanda por el desarrollo humano y el carácter instrumentalista que da origen al currículo por competencias.

Curricular changes in Chilean higher education in the last 15 years have not been harmoniously accompanied by a transformation of university teaching. The discrepancy between the curriculum and the actual teaching has become an obstacle for the results that these curricular innovations seek. Thus, the present article enlists the foundations of formative methodological strategy for teaching, focused on the development of competencies. This will allow an actual change in teaching practices, so that these curricular innovations can gain consistency and better respond to the challenges that gave rise to them. Furthermore, we formulate a theoretical-practical articulation that supports a new way of teaching in coherence with curricular innovations, and at the same time, addresses the existing tension in the training process of university professionals between the demand for human development and the instrumentalist character that gives rise to the competency curriculum.

Keywords

College teaching

Higher education

Pedagogical practice

Teacher competencies

Curriculum by

competencies

Recepción: 29 de septiembre de 2020 | Aceptación: 26 de mayo de 2021

DOI: https://doi.org/10.22201/iisue.24486167e.2022.175.60178

* Académica de la Facultad de Filosofía y Educación de la Universidad Metropolitana de Ciencias de la Educación (UMCE) (Chile). Doctora en Ciencias de la Educación. Líneas de investigación: hermenéutica e investigación educacional; hermenéutica y metodología en la formación de profesores. CE: teresa.rios@umce.cl 
Los grandes cambios habidos en la educación superior chilena en los últimos 30 años han traído aparejados nuevos y grandes desafíos al sistema. Entre el cúmulo de transformaciones que han impactado destacan dos con especial repercusión en el ámbito de la docencia: la primera de ellas se refiere a la masificación de la matrícula de pregrado (Akalu, 2016; Brunner, 2013; Larotonda, 2018), que en el caso de Chile pasó de $132 \mathrm{mil}$ al inicio de los gobiernos post-dictadura (año 1990), a 655 mil en el año 2020 (CNED). ${ }^{1}$ Esta situación ha traído consecuencias económicas, sociales y culturales que impactan a los procesos de formación de profesionales (Linne, 2018; Soto 2016; Larotonda, 2019), sobre todo porque este aumento significó el ingreso a las instituciones de educación superior de jóvenes de sectores que nunca antes lo habían podido hacer. En el caso chileno, este proceso se dio de manera atrasada con respecto a otras partes del mundo, lo que dio origen a un nuevo tipo de estudiante, denominado genéricamente "estudiante de primera generación", que resulta mayoritario en la mayor parte del sistema (Castillo y Cabezas, 2010). La segunda transformación se refiere a las profundas innovaciones curriculares de los últimos 15 años, en parte como respuesta al cambio recién expuesto, pero sobre todo como parte del proceso global impulsado a partir del Proyecto Tuning América Latina (Vélez et al., 2018; Muñoz y Sobrero, 2006), que significó la adopción de diversas variantes de modelos curriculares basados en el desarrollo de competencias.

Estas transformaciones, que han significado un profundo cambio en las casas de estudio, no han sido acompañadas por transformacio- nes de la misma proporción en las prácticas docentes, de manera que se guarde la debida coherencia entre el modelo curricular (por competencias) y el perfil de los nuevos estudiantes. En efecto, la literatura sobre la temática (De Juanas y Beltrán, 2014; Larotonda, 2018; López Pérez, 2018; Tejada, 2018) da cuenta de que muchas veces los profesores siguen reproduciendo formas de hacer docencia originadas dentro de modelos curriculares anteriores, centrados en la trasmisión de contenidos y pensados para un tipo de estudiantes altamente seleccionados a los que Bourdieu y Passeron (2009) denominaron como los "herederos", y que actualmente sólo se encuentran en un grupo muy reducido de casas de estudio de Chile. El resultado de esta situación es una previsible discordancia entre las prácticas pedagógicas y las que requerirían los nuevos perfiles de estudiante, así como un desajuste entre aquéllas y las que supone el nuevo modelo curricular.

Así, resulta de interés proponer y fundamentar estrategias que contribuyan a la transformación de las prácticas docentes, de manera que las innovaciones curriculares ganen en consistencia y respondan mejor a los desafíos que les dieron origen. Se presenta, pues, una estrategia metodológica formativa para una docencia en la educación superior con sus fundamentos, que nacen de las experiencias de aplicación en dos instituciones en dos momentos diferentes. ${ }^{2}$ Está enfocada en el desarrollo de competencias, y en el supuesto de que este cambio sólo se podrá dar cuando los/as docentes tomen conciencia y conocimiento de los fundamentos sobre los cuales sostienen sus prácticas, para así fortalecerlas y/o transformarlas desde el saber académico. Se trata de un cambio que se constituye, en términos curriculares, en lo

\footnotetext{
${ }^{1}$ Es de notar también el aumento en los últimos nueve años en la matrícula en los institutos profesionales, los que pasaron de tener 122 mil 500 estudiantes en 2005 a 363 mil este 2020, es decir, un aumento de 300 por ciento en 15 años. La matrícula total del sistema alcanzó en 2020, un millón 144 mil estudiantes. Sin embargo, es de destacar que la tasa de crecimiento presenta una marcada desaceleración el último quinquenio, con leve decrecimiento los últimos dos.

2 Esta estrategia fue aplicada por el CICAD de la UTAL (Universidad de Talca, Chile) el año 2007-2008 y se está despliega actualmente en la UMCE (Universidad Metropolitana de Ciencias de la Educación, Chile), institución que financia el trabajo de la autora principal de esta publicación a través del Proyecto "Red Interuniversitaria de Cobertura Nacional para el Mejoramiento de la Calidad de la Formación de Pregrado mediante la incorporación institucional del diseño curricular en base a competencias RINAC” (AUS 0402).
} 
que se ha llamado un giro paradigmático (Rueda Beltrán et al., 2019; Tejada, 2018), pues supone la transformación de los supuestos que sostuvieron la docencia en la educación superior por mucho tiempo, y en la cual fueron formados la mayoría de los actuales profesores.

La propuesta metodológica comprende dos dimensiones de la práctica de la docencia a través de las cuales los profesores podrán concretar el giro antedicho: la reflexiva y la fundante. La primera propicia la "toma de conciencia”, que consiste en traer a la luz aquellas ideas y creencias que han dado origen a sus actuales prácticas docentes, mismas que tienden a reproducir tanto el modo en que el/la docente aprendió, como los parámetros conceptuales institucionales que la guiaron hasta la actualidad (Bourdieu y Passeron, 2009; Boudon, 2003). La dimensión fundante, por su parte, cimienta y estructura un acercamiento comprensivo al fenómeno de la formación universitaria e integra los aportes tradicionales de la didáctica (y de la psicología cognitiva aplicada a ésta) con los del mundo de las humanidades, lo que permite conjugar el aspecto instrumental del saber didáctico con la dimensión de sentido que tiene la formación universitaria.

Se estima que la conjunción de estas dos dimensiones en los procesos de formación permite iniciar el tránsito desde un/a "docente competente", capaz de aplicar eficazmente técnicas didácticas asociadas a las nuevas exigencias curriculares, a un/a "docente reflexivo/a transformador/a", como lo denominan las tradiciones de raíz crítica (Freire y Shor, 2014; Schön, 1992; Zeichner y Liston, 1993); ser competente significa que comprende los fundamentos y los principios que sustentan el cambio requerido, lo que le permite resignificar $y$ dotar de sentido a la enseñanza y a las técnicas didácticas que utiliza. En efecto, aprender las técnicas propias de una docencia orientada al desarrollo de competencias aporta a la formación profesional, pero apropiarse de la lógica que hay detrás de ese proceso contribuye al desarrollo de la iniciativa, la creación y producción de nuevos aprendizajes que movilizan las competencias desarrolladas en los estudiantes en pro de una transformación real.

Con base en lo anterior, la estrategia metodológica que se presenta asume el desafío de formular una articulación teórico-práctica que dé sostén a un nuevo modo de enseñanza que resuelva la discordancia planteada entre currículo y docencia y que, al mismo tiempo, atienda la tensión que se produce en el proceso de formación de un profesional universitario entre la demanda por el desarrollo humano y el carácter instrumentalista que da origen al currículo por competencias.

\section{NUEVAS TENSIONES EN LA EDUCACIÓN SUPERIOR E IMPACTO EN LA DOCENCIA}

Como ya se señaló, los sistemas universitarios han sufrido en los últimos años un proceso de masificación de su matrícula que ha tenido una serie de consecuencias sobre las casas de estudio al cuestionar el carácter elitista vigente hasta hace poco tiempo. Esto ha generado nuevas tensiones (Brunner, 2013; Bernasconi, 2014; Akalu, 2016) entre las que se destacan dos: la primera se da entre lo que puede denominarse como "visión elitista" de la universidad, que se enfrenta a la "visión centrada en el derecho"; es decir, entre la que entiende que las universidades son el espacio donde se forman las elites intelectuales (Brunner, 2013) a partir del capital cultural de origen de los estudiantes (Bourdieu y Passeron, 2009), versus la que entiende a la educación superior como un bien social (De Sousa Santos, 2005; Giroux, 2016). En esta primera tensión se contraponen propuestas curriculares basadas en rigurosos sistemas de selección para el ingreso y prácticas docentes que tienden a someter a prueba a los estudiantes, versus prácticas curriculares y docentes que procuran brindar condiciones básicas para el desarrollo adecuado de las trayectorias académicas.

Una segunda tensión aparece a partir de la irrupción delos principiosfilosóficos propios del neoliberalismo que sirvieron de fundamento de 
las reformas universitarias en Chile en la década de 1980, reforma que promovió la masificación y privatización de la educación superior, como bien señalan Vidal et al. (2017). La tensión se da aquí en torno a la concepción de la "educación con un fin público" (aunque elitista, como ya se presentó), contra la que entiende a la "educación como un bien de consumo", es decir, un producto que no tiene un fin social, sino la satisfacción de necesidades individuales, en la que interesa el aumento de la escala, la eficiencia de la "producción” y la calidad del producto. En ésta, el/la estudiante se transforma, poco a poco, en un/a cliente que "compra" una capacitación o certificación (Vergara, 2012).

Estas tensiones han tenido consecuencias en la estructura del sistema pues, como se ha señalado, el proceso de masificación estuvo marcado no sólo por el aumento de la matrícula, sino también por su progresiva privatización (Del Valle et al., 2017; Vidal et al., 2017), lo que ha afectado, a su vez, a las relaciones entre profesores y estudiantes, y entre profesores e instituciones. En efecto, los requerimientos de cambios en las relaciones de los actores educativos nacen mayoritariamente de la necesidad de responder a órganos de control externos para mostrar "eficiencia" en el uso de los recursos frente a esas instancias, y no tanto para tratar de construir condiciones para el desarrollo académico de aquellos estudiantes que tradicionalmente no ingresaban a la educación superior (los estudiantes de primera generación). Así, sea porque se entiende que estos cambios ameritan en justicia una transformación de la docencia universitaria que democratice los procesos de formación, o por la sola búsqueda de un funcionamiento eficiente y eficaz de los procesos de capacitación y certificación, hoy se exige a los profesores que funden su enseñanza en un nuevo currículo, que procuren el desarrollo de competencias profesionales en sus estudiantes y que abandonen las típicas prácticas del "colador".

Por otra parte, la adopción de currículos basados en el desarrollo de competencias (o por competencias) han representado una tensión para las instituciones, especialmente para los/las profesores/as. Cabe recordar que una de las características centrales de este tipo de currículos es que están orientados a los resultados y, por ello, organizan sus esfuerzos formativos hacia el logro de compromisos de desempeño (Morke et al., 2013); esto significa un cambio sustantivo del mundo académico. En efecto, los/as docentes universitarios fueron mayoritariamente formados y ejercieron su docencia por mucho tiempo dentro de la lógica de currículos por objetivos, en donde el foco o centro del proceso formativo no está en los resultados de aprendizaje de los estudiantes, sino en el cumplimiento de objetivos; esto trae implícito un cierto rol docente que supone la "entrega" de contenidos para que sean aprendidos por los estudiantes (el rol del estudiante). En contraste, el currículo basado en el desarrollo de competencias, desde una perspectiva constructivista, entiende que el docente guía o media un proceso de formación profesional en el que los/as estudiantes desarrollan capacidades y movilizan atributos y saberes para alcanzar nuevos resultados de aprendizaje. Éstos, por su parte, evidenciarían el crecimiento en los niveles de desarrollo de las competencias profesionales (Tardif, 2003; 2009), lo que le permitirían al egresado actuar de forma eficaz en situaciones problemáticas propias de su quehacer profesional (Le Boterf, 2001).

Esta diferencia hace necesario que los/as profesores/as, ya expertos en sus conocimientos disciplinares, realicen un esfuerzo por "reenmarcar" (Schön, 1992) su enseñanza al orientarla hacia la gestión y logro de tales compromisos formativos (Le Boterf, 2018). Aparece, entonces, la necesidad tanto de comprender mejor y de resignificar (contextualizándolo) el modelo curricular mismo (Ríos, 2008), como de realizar esfuerzos que promuevan cambios reales en las prácticas docentes. Se trata de una tarea compleja, pues la docencia universitaria, como ya afirmaba Emilio Tenti en los años noventa, es una red de múltiples 
entrecruzamientos que implican el conocimiento, la educación, la ciencia, el arte, la verdad, la política, la ética, el trabajo, la profesión, la enseñanza, la experticia, la técnica, la teoría y la práctica; claramente su análisis y ejercicio no es una tarea unidimensional (Tenti, 1993).

La estrategia metodológica que aquí se plantea apunta a lograr coherencia entre los distintos elementos que se ponen en juego en la práctica docente a partir del cambio de esquemas en la forma de hacer docencia, misma que suele reproducir las lógicas del currículo tradicional. En este contexto de cambio y problema aún no resuelto, el/la profesor/a universitario se encuentra con la exigencia de dar un vuelco en la comprensión y la práctica docente.

Para lograr dicho vuelco se espera que los/as docentes, en tanto que sujetos de un nuevo aprendizaje, se respondan a sí mismos el interrogante de cómo ser profesor desde la nueva lógica, al tiempo que respetan e incorporan tanto su experiencia como el conocimiento que han ido construyendo en sus prácticas (Freire y Shor, 2014; Schön, 1992; Zeichnner, 1993).

Es así como la estrategia supone, junto con el desarrollo de la capacidad reflexiva, un cambio que respete e integre la experiencia de los/ as docentes para que, dentro de este marco, se apropien de elementos conceptuales y procedimentales del nuevo modelo, de manera que puedan identificar la dimensión constructiva que éste posee. Si bien este modelo ha nacido dentro de una racionalidad técnica (Macchiarola, 2007), se espera poder instrumentalizar sus conceptos en pos del desarrollo de las capacidades reflexivas de docentes y estudiantes, lo que permitiría dar un giro al rol del profesor universitario que va desde el aplicador de técnicas para desarrollar competencias profesionales, hacia el profesor reflexivo transformador.

Otro de los problemas de la educación superior en este contexto de cambio ha sido la fragmentación de los saberes que componen el proceso de formación, potenciada, entre otras razones, por una docencia orientada hacia la reproducción y a la tendencia tradicional de que cada disciplina tenga un fin en sí misma. Paradójicamente, una de las fortalezas que podemos encontrar en esta nueva propuesta es la posibilidad de romper con las prácticas que tienden a la reproducción al ampliar el proceso de aprendizaje más allá de la mera retención de contenidos, así como al exigir que se produzca algo nuevo y diferente a partir de los contenidos propios de cada disciplina, como se mostrará más adelante (Perrenaud, 2008). Esto es posible debido a la nueva orientación que deben alcanzar las actividades curriculares que componen la malla de las carreras; en efecto, dado que se cuenta con un perfil de egreso como horizonte común es posible armonizar los procesos de formación y potenciar desde las aulas el desarrollo de habilidades, capacidades, atributos y conocimientos; al movilizar todo ello se abre paso a un proceso de desarrollo de niveles de competencias y se avanza en la concreción de dicho perfil.

\section{LA ESTRATEgIA METODOLÓGICA}

La propuesta tiene como objetivo el desarrollo de las competencias docentes de los profesores universitarios de manera armónica (o en concordancia), con los requerimientos que los nuevos currículos basados en el desarrollo de competencias plantean, al tiempo que aborda las tensiones a las cuales estos currículos pretenden dar solución y que ya fueron expuestas. Dicho desarrollo, como se señaló, está orientado hacia un giro paradigmático de la enseñanza. Para ello se propone una metodología que, al trascender la simple capacitación, se constituya en un verdadero proceso formativo que transforme la mirada sobre la enseñanza, de manera que los cambios que en ésta sucedan radiquen en una verdadera apropiación reflexiva de la labor, y no en una mera ejecución de "técnicas". Esta transformación en la práctica docente supone conservar y cultivar el tradicional saber disciplinar de los académicos, pues éste es el factor que permite proyectar el cambio de manera fundada. 
Este planteamiento supone que el aprendizaje se da en un proceso que contempla tres momentos: anclaje, apropiación y producción, que se llevan a cabo en el transcurso de una cierta unidad de aprendizaje. No deben ser confundidos con momentos de una clase en particular (el típico inicio-desarrollo-cierre), sino que hacen referencia a grandes entidades de aprendizaje, ya que la estrategia entiende los procesos de enseñanza-aprendizaje desde una lógica molar, alejada de las propuestas que los atomizan. El tradicional ordenamiento de las asignaturas en unidades de aprendizaje recoge este posicionamiento epistémico, aunque para los efectos de la estrategia metodológica la unidad no necesariamente está determinada por los programas. Incluso, en casos como cursos cortos, esta unidad podría ser la actividad curricular misma.
Cada uno de los momentos señalados (anclaje, apropiación y producción) da cuenta de una progresión en el proceso de aprendizaje, por lo que las actividades del/a docente tendrán propósitos específicos en consonancia con dichos momentos. Esta progresión inicia con una anamnesis sobre conocimientos y experiencias pasadas que se conectan con el contenido abordado en la unidad de aprendizaje (anclaje), para luego vincularlos con los contenidos y habilidades que se pretenden desarrollar en la unidad (apropiación), y finalmente dar un espacio en donde, a partir de la apropiación de dichos contenidos o saberes, el sujeto que está en situación de aprendizaje genere productos en los que movilice y recree dichos aprendizajes (producción), proyectándolos, para hacer posible, de esta manera, la evaluación del proceso total. Estos momentos se pueden sintetizar en el esquema siguiente.

\section{Figura 1. Momentos de la estrategia metodológica}

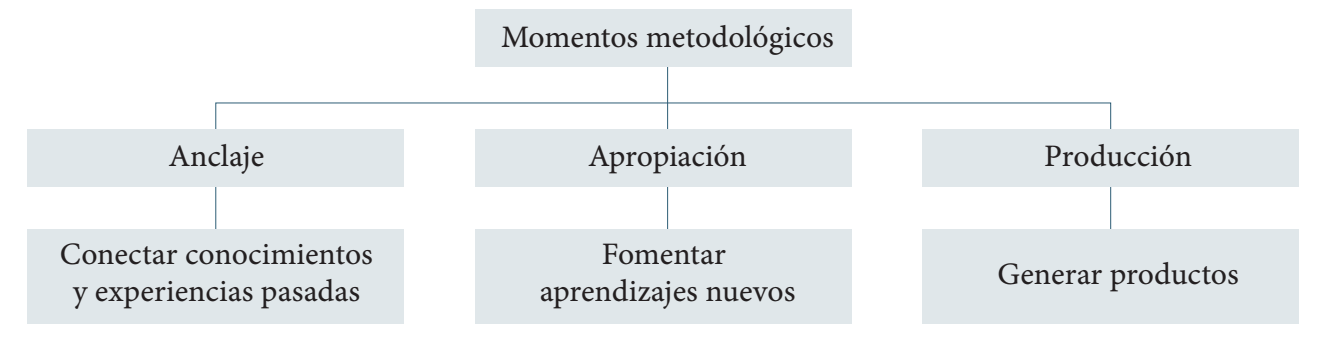

Fuente: elaboración propia.

El conjunto de los momentos apunta a alcanzar el resultado de aprendizaje esperado. En el caso de los estudiantes de carreras universitarias, se orienta al desarrollo progresivo de las capacidades que irán aportando a la construcción de la competencia o resultado de aprendizaje comprometido en el programa y en el perfil de egreso de cada carrera. Para el caso de profesores/as que se encuentran en proceso de formación o perfeccionamiento (cuya estrategia se está presentando aquí) se dirigen al desarrollo de las competencias involucradas en la docencia universitaria en coherencia con las innovaciones curriculares.

Tanto los momentos, como las técnicas en que se operacionalizan, se fundan en una vinculación teórica entre dos grandes conjuntos de elementos: los primeros son tributarios del constructivismo sociohistórico y de la psicología cognitiva, y sostienen que el conocimiento y el aprendizaje son principalmente un producto de la interacción social (Vygotsky, 1985; Ausubel et al., 1983). Los segundos se nutren de los desarrollos de la fenomenología 
(Schutz, 2003; Berger y Luckmann, 2001) y de la hermenéutica reflexiva (Ricoeur, 2001, 2008; Ríos, 2004a, 2004b, 2008, 2013). Esta articulación apunta, como ya se señaló, a conjugar el aspecto técnico instrumental de la didáctica con el formativo, propio de las ciencias sociales y las humanidades.

Teniendo presente lo expuesto hasta el momento, la estrategia metodológica que se presenta pretende que los/as docentes se apropien de esta manera de entender el proceso de formación y que luego ellos/as desarrollen su actividad docente según estos principios. Por esta razón, para que puedan incorporar el nuevo modelo se propone que lo aprendan y comprendan a través de un proceso que utilice estos mismos tres momentos.

Se presenta, entonces, en qué consiste cada uno de los tres momentos de aprendizaje, en conjunto con la propuesta de formación de profesores/as que se deriva de las experiencias de aplicación ya hechas. Es decir, el detalle de cada etapa se despliega en conjunto con la propuesta metodológica de formación de los docentes para atender a los nuevos requerimientos en la educación superior.

\section{Primer momento: anclaje}

Las corrientes constructivistas sostienen que los aprendizajes se erigen a partir de la conexión de lo nuevo con estructuras ya establecidas en el sujeto, lo que le permite asimilarlo (en términos piagetianos) o apropiarse de él (en términos vigotskianos). Estas estructuras son dinámicas, evolucionan y se transforman; se construyen y reconstruyen de manera modular en el proceso de aprendizaje, tal como lo señala Bruner (1972) cuando habla de "andamiaje".

Con base en lo dicho, el momento del anclaje tiene el propósito de unir o asegurar los nuevos aprendizajes a uno sólido, ya estructurado; es decir, vincularlo a las estructuras ya desarrolladas. De esta forma, y a partir del carácter configurador del acto de narrar (Ricoeur, 2001; 2008) en conjunto con el fecundo concepto vigotskiano de "zona de desarrollo próximo"
(Vygotsky, 1985), este momento apunta a que el docente en formación, con la ayuda de un profesor o profesora experto, conecte a través de un relato las experiencias que ha reconocido como buenas o positivas recientemente con los nuevos conocimientos que surgen de las propuestas nacidas de las reformas curriculares y los enfoques en que ellas se fundan. Una vez hecha esta conexión, se hace necesario una toma de distancia para analizar y reinterpretar (Schutz, 2003; Schön, 1992) lo narrado desde la mirada que los nuevos enfoques curriculares plantean, lo que implica un esfuerzo por salir del paradigma del cumplimiento de objetivos para entrar en el desarrollo de competencias.

Como puede verse, para lograr este propósito es necesario trascender lo estrictamente didáctico (dimensión instrumental) y fundarlo en los aportes de las humanidades (dimensión de sentido). Por ello, el "relato" ocupa un lugar fundamental desde el plano metodológico, entendido desde la tradición hermenéutica crítica (Ricoeur, 2001; 2008). Desde este "relatar" los/as docentes podrán resignificar las experiencias narradas y establecer una conexión entre la experiencia docente pasada con una proyección (transformada) en el futuro (Ricoeur, 2001; 2008) que recoja los requerimientos de los nuevos enfoques curriculares.

Es importante señalar que la idea de recurrir al relato como modo de recrear la experiencia docente se sustenta en los principios de la hermenéutica crítica de Paul Ricoeur (2001; 2008), según la cual el relato tiene una propiedad de configuración, lo que es coherente con lo que se pretende en la etapa de anclaje. En efecto, cuando alguien narra, compone una historia y crea un espacio entre lo vivido y la historia relatada. Según esta premisa, el/la docente, al explicar su experiencia en la relación de diálogo con un/a profesor/a experto/a, se comprende a sí mismo/a. Esto es posible en tanto que el relato está íntimamente relacionado con la temporalidad, de manera que marca, articula y clarifica (Ricoeur 2001; Ríos, 2013) la experiencia narrada. 
Desde esta perspectiva se puede deducir que las experiencias obtenidas por los docentes en las clases que ya han impartido, de la modalidad que sean (clases magistrales, actividades prácticas de trabajo personal y en equipo, etc.), en el acto de ser narradas crean una conexión con una dimensión personal y humana que es importante rescatar, puesto que al mismo tiempo se producen las condiciones para articular estas experiencias con todas aquellas que podrá desarrollar en un futuro. Se recurre aquí a la afirmación de Ricoeur (2001: 18), que dice que "entre el vivir y el relatar se abre un espacio que, por ínfimo que sea, permite el descubrimiento de una experiencia viva"; esto plantea la posibilidad de que, al tiempo de relatar esa experiencia, el/la docente tome distancia de ella, de manera que pueda analizarla y comprenderla dentro de una lógica diferente a la que habitualmente acostumbraba a seguir.

$\mathrm{El} / \mathrm{la}$ docente tendrá que distanciarse de sus propias experiencias relatadas y reflexionarlas; este paso implica el ejercicio de mirarse a sí mismo/a como si fuera otro, no dentro de la lógica terapéutica, sino desde un acto existencial que permite focalizar la narración de sus experiencias para recrearlas y comprenderlas en el nuevo contexto, con el fin de configurar un nuevo proceso comprensivo de su quehacer.

Además de mirarse a sí mismo/a, en esta etapa el/la docente deberá tomar conciencia del contexto en el que se desenvuelve para comprenderlo de un modo nuevo, y para lograrlo se recurre a la noción de "actitud desinteresada”, proveniente de la sociología fenomenológica de Schutz (2003; Osorio,1999). Este concepto refiere a un modo de acercamiento a la realidad que se caracteriza por la suspensión temporal de los juicios y redes de referencia que han orientado la acción; es así como se puede tomar distancia de la realidad y se hace posible reflexionar sobre ella. Es decir, quien toma una actitud desinteresada deja de vivir dentro del marco incuestionado desde el que orientaba su acción docente y decide observar ese marco con ojos nuevos (Schutz, 2003), con la consistencia que le permite la distancia intencional asumida. Así, el/la docente en esta etapa intentará "no situarse ni situar su propia condición de interés como centro... [de su quehacer], sino adoptar otro origen de coordenadas" (Schutz, 2003: 141) para orientar la transformación de su acción. Esta decisión implica "poner entre paréntesis el mundo narrado y suspender los juicios que tiene con respecto a éste... de manera de volver discutible aquello que antes era cierto y evidente para él" (Ríos, 2005: 63), así como convertir su experiencia docente - que hasta ahora había sido válida e incuestionable - en un simple fenómeno.

En este escenario de toma de distancia surge en el/la docente un conflicto cognitivo (Piaget, 1999) que abre el espacio para que pueda darse cuenta de que si el protagonismo del aprendizaje debe estar, ahora, en el estudiante, y si es en éste en quien recae la responsabilidad de obtener, movilizar y producir contenidos, así como desarrollar las habilidades que lo hagan posible, entonces el rol del docente debe centrarse en propiciar el clima adecuado, evocar los conocimientos previos, planificar la progresión de las acciones que permiten que un/a estudiante alcance el desarrollo esperado de la competencia y, sobre todo, diseñar las estrategias evaluativas que permitan dar cuenta de ese salto.

Se trata, pues, de que el/la profesor/a comprenda el desplazamiento que implica este hecho en el rol docente. En las actuales circunstancias, además de ser un especialista en su disciplina el/la profesor/a requerirá de competencias docentes, es decir, aquéllas que le posibiliten conducir el recorrido que debe hacer un/a estudiante para lograr el desarrollo de conocimientos, habilidades, capacidades, destrezas y atributos que supone el nuevo modelo. Así llegará a entender profundamente que su rol ha cambiado: hoy se espera que facilite y sea capaz de mediar esta trayectoria, y para ello necesitará nuevos recursos y un nuevo conocimiento. 


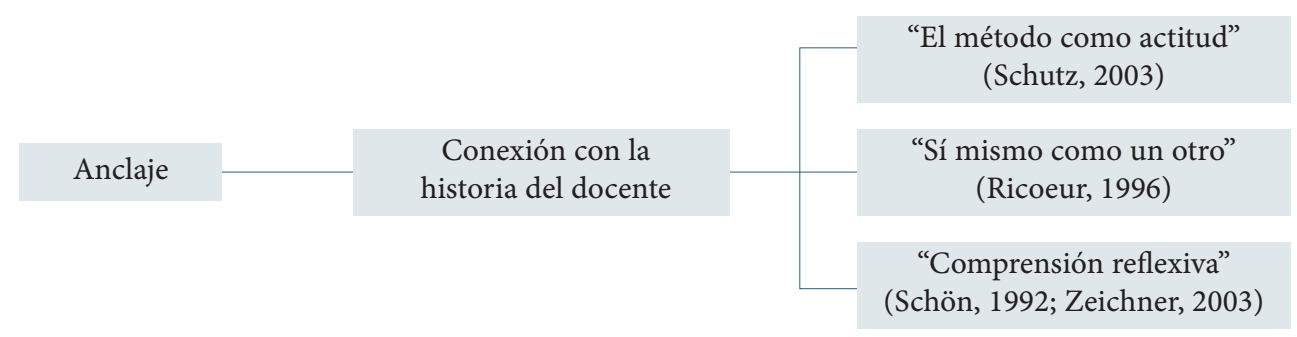

Fuente: elaboración propia.

La Fig. 2 sintetiza la primera fase del proceso de transformación de la docencia, que hemos llamado anclaje.

\section{Segundo momento: la apropiación}

El proceso desarrollado en la fase de anclaje deja al docente en condiciones para integrar los nuevos conocimientos que requiere la aplicación del nuevo modelo y las técnicas asociadas a él. En este segundo momento metodológico, llamado fase de apropiación, se acercará a los nuevos conocimientos (contenidos) necesarios para el desarrollo de los resultados de aprendizaje comprometidos en el proceso formativo, lo que supone la apropiación del sentido y las fundamentaciones sobre las cuales se sustentan las técnicas propias de esta etapa. Esta apropiación conduce a una toma de conciencia sobre el cambio en la lógica sobre la cual se sustenta la docencia dentro de los nuevos currículos universitarios; es decir, permite un acto metacognitivo (Bruner, 1972) o de insight (Lonergan,1992).

Este proceso vivido por el/la profesor/a es el que le permitirá realizar, en su propia docencia, el acercamiento a los nuevos contenidos involucrados en una unidad de aprendizaje. Esto se realiza a través de la aplicación de técnicas orientadas hacia este fin, tales como clases expositivas, trabajos de investigación guiados por profesores, lecturas guiadas y trabajos de síntesis a través de esquemas gráficos (mapas conceptuales, mapas mentales, etc.), entre otras.

Este momento metodológico supone enfrentar un doble desafío: por un lado, desarro- llar la capacidad reflexiva en sus estudiantes sobre los tópicos a aprender y, por otro, promover la capacidad crítica sobre los conocimientos que se esperan desarrollar. Como se señaló en la etapa anterior, esto requiere de la mediación de un profesor/guía experto/a que apoye la construcción de nuevos aprendizajes. En este proceso al principio el apoyo es grande y poco a poco se va retirando hasta que se logra de manera autónoma (Bruner, 1972). De este modo, el/la profesor/a podrá desarrollar en la planificación de sus propias clases los procesos metacognitivos que le permitan a sus estudiantes trascender los aprendizajes de tipo memorístico y resignificarlos y orientarlos al desarrollo de las competencias comprometidas.

En este sentido, la teoría histórico-cultural enfatiza el contexto social en el cual se hace posible la apropiación de los contenidos y de las competencias y habilidades del individuo asociadas a ellos, para que los sujetos del aprendizaje puedan ser conscientes de los procesos psicológicos involucrados en la tarea y, además, puedan autorregularse. De este modo, la metacognición - entendida como los pensamientos voluntarios y conscientes que tienen como objeto otros pensamientos-, en tanto que conscientes y voluntarios no solamente son potencialmente controlables por la persona que los experimenta, sino también potencialmente comunicables de forma que sean accesibles a otros (Guerra, 2003).

Una vez desarrollados los aprendizajes de los contenidos involucrados es el momento de proponer las formas de evaluarlos a través 
Figura 3. Segundo momento: la apropiación

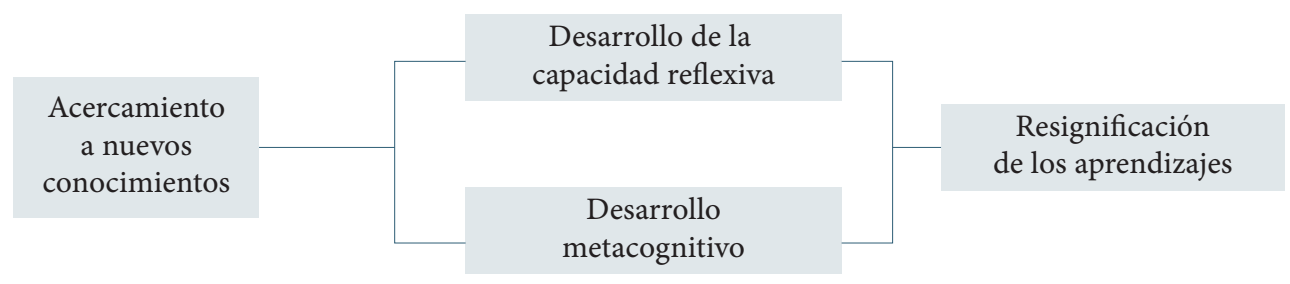

Fuente: elaboración propia.

de técnicas de índole tradicional, tales como controles, ensayos, pruebas de desarrollo y de alternativas, resolución de problemas y otros. La Fig. 3 presenta los pasos que dará el docente en esta etapa.

Esta fase recurre a las actividades propias de la docencia tradicional en las que en un primer momento se generan las instancias de acercamiento a los nuevos contenidos (clases expositivas, trabajos de investigación, etc.), para luego realizar una evaluación sobre el aprendizaje de dichos contenidos (pruebas, resúmenes gráficos tales como mapas conceptuales, pruebas de desarrollo, resolución de problemas, etc.). Es decir, se trata de enmarcar las actividades tradicionalmente realizadas en la docencia en un proceso mayor, precedidas de la etapa de anclaje en la que los sujetos del aprendizaje se disponen y hacen presentes las estructuras que les permiten apropiarse de los nuevos saberes, para que, finalmente, estos nuevos saberes sean proyectados en la tercera etapa, la de "producción".

Desde esta perspectiva, el cambio en la docencia no pasa por reemplazar lo que ya se hace, sino por enmarcarlo en una estructura que le dé sentido y lo oriente hacia los fines que pretenden desarrollar los nuevos currículos.

\section{Tercer momento: la producción}

La tercera fase se caracteriza por ser el espacio en que los aprendizajes adquiridos en las fases anteriores se proyectan en la creación, y no en la reproducción. En línea con la tradición del constructivismo sociohistórico, la "apropiación" supone una transformación en lo aprendido (Piaget, 1999; Leóntiev, 1985; Vygotsky, 1985), por lo que el proceso de aprendizaje debe finalizar en un momento de expresión y/o proyección de esta "transformación" la cual, a su vez, se constituye en una etapa evaluativa, entendida como constituyente del proceso de enseñanza-aprendizaje (Santos, 2003). Lo anterior implica, claramente, un cambio en la concepción y función tradicional de la evaluación.

Uno de los problemas de los enfoques curriculares tradicionales es que asumen que el proceso de aprendizaje finaliza en un acto evaluativo centrado en reproducir o devolver el conocimiento. Desde la lógica de la estrategia metodológica que se presenta, la evaluación debería ser un acto de producción, expresado en la elaboración de algo nuevo a partir de la transformación de lo aprendido. Se trata, pues, de un modo de evaluación que trasciende a las ideas tradicionales y se centra en la búsqueda de constatación del nivel de desarrollo de las competencias comprometidas (Tardiff, 2003).

En esta etapa el/la docente toma decisiones metodológico-didácticas porque selecciona y/o crea dispositivos de evaluación del desempeño de sus estudiantes en los cuales éstos puedan expresar los logros obtenidos con la movilización y creación de algo nuevo. De esta forma se desarrolla la capacidad de proyectar, dado que se aplican técnicas cuyo fin es movilizar los conocimientos, actitudes y capacidades en pos de la creación de dispositivos que permitan solucionar problemas reales u abstractos desde los saberes y dominios 
de aplicación de la disciplina que se imparte. Las técnicas más apropiadas para este fin son las que se basan en la metodología de la resolución de problemas o de proyectos, en algunas de sus variantes, ya sean de intervención social y/o educativa, de producción de prototipos o pilotos, o proyectos de investigación, entre otros, según la disciplina en cuestión.

La fase de la producción se constituye, así, en la realización del aprendizaje cuando el/la que aprende da un paso en su autonomía y moviliza, transforma y produce conocimientos concretizados en un producto nuevo. En el caso de los docentes en formación, el cumplimiento de esta fase se despliega en un proceso que comienza con la aplicación de las técnicas de evaluación recién mencionadas, derivadas del proceso de proyección de los aprendizajes, para luego comprender - en un acto metacognitvo-cuál es el recorrido total de su propio proceso de aprendizaje, entendido desde esta propuesta metodológica. Esto se concretiza en la programación de una unidad de alguna de las asignaturas que dicta, siguiendo esta estrategia. Es así como, junto con posibilitar a los/as profesores/as en formación la proyección propiciada en esta última fase, se logra su habilitación para volver a hacerlo en el ejercicio de su rol docente; de esta manera construyen un nuevo modo de hacer docencia, y comprenden las limitaciones que tenía su anterior forma de enseñar.

\section{REFLEXIONES FINALES,}

\section{A MODO DE CONCLUSIÓN}

Este trabajo busca explicitar cómo la estrategia didáctica presentada puede aportar a la resolución de las tensiones descritas y fortalecer la coherencia curricular al alinear la docencia con los principios de los nuevos currículos. Al hacerlo, se resuelve la aparente contradicción entre el carácter tecnológico instrumentalista que dio origen al currículo por competencias y la demanda por el desarrollo humano en los procesos de formación de profesionales.
En efecto, el modelo curricular basado en competencias, al articularse con los proyectos universitarios que han tenido tradicionalmente un carácter humanista, profundiza esta tensión. Para superarla es necesario interpretar la propuesta curricular por competencias a la luz de la tradición de los proyectos educativos institucionales, evitando las miradas totalizadoras que procuran su instalación sin abrir un proceso de aprendizaje reflexivo en torno a sus fortalezas y debilidades. De este modo se podrá convertir en un instrumento estratégico para mejorar la formación de profesionales competentes, transformadores y críticos que respondan a los requerimientos de la sociedad actual.

La propuesta metodológica pretende ser una vía de encuentro que termine con esta tensión. Para este propósito se recurrió a los aportes del constructivismo, la fenomenología y la hermenéutica, que son los que permiten una articulación entre la necesidad de formar un profesional competente para actuar de forma eficaz en todos los ámbitos de desenvolvimiento, especialmente en el laboral, y la de formar personas que, comprometidas con la realidad, sean capaces de proponer y desplegar proyectos profesionales orientados al desarrollo humano.

El nuevo modelo curricular plantea ciertos desplazamientos que preocupan a los docentes, puesto que los sacan del marco de lo que ha constituido su experiencia. El protagonismo del docente se desplaza al estudiante, la preeminencia de los contenidos hacia el desarrollo de competencias, y la importancia de los resultados se corre hacia la generación de productos. Esto hace que el rol del docente universitario exija, además del saber intelectual, un saber de tipo didáctico. Lo anterior provoca que el/la docente tienda a resistirse a este nuevo requerimiento, puesto que trae consigo, por una parte, la necesidad de aprender técnicas nuevas independientemente de que considere necesario adoptarlas y, por otra, la aceptación de un concepto ("competencias") cuyo significado no 
está claro y que procede de un sector ajeno a la tradición de la educación superior.

La propuesta metodológica aquí planteada se asienta en un sustento teórico que explicita los principios de las tradiciones de la educación superior y hace posible que se supere la resistencia del/a docente a adoptar el modelo por competencias, puesto que le invita a constituir una trayectoria que implica reflexionar y transformar (no reemplazar) sus prácticas a partir de una experiencia configuradora de sentidos; a ser gestor/a de su propio cambio en un ejercicio de autonomía y creatividad que posibilita, además, la construcción colectiva de un nuevo modo de concebir la enseñanza.

Se trata de asumir el desafío de articular, a través de la docencia, las necesidades del mundo laboral y el mundo social a partir del desarrollo humano (Sen, 2000), desde una comprensión del escenario y del sentido que plantea el nuevo modelo, y desde la resignificación del concepto "desarrollo de competencias". Así se podrá formar a las nuevas generaciones en un perfil profesional que mantenga el necesario equilibrio entre las necesidades que dieron origen al currículo por competencias y un proyecto social basado en el crecimiento humano.

En este sentido, el cambio se traduce en el paso de un docente conocedor experto de una disciplina, a un docente que reflexiona e interpreta su saber y lo vincula con los intereses y proyecciones de sus estudiantes situados en un contexto social real.

\section{REFERENCIAS}

AкAlu, Girmau Abebe (2016), "Higher Education 'Massification' and Challenges to the Professoriate: Do academics' conceptions of quality matter?", Quality in Higher Education, vol. 22, núm. 3, pp. 260-276. DOI: https://doi. org/10.1080/13538322.2016.1266230

Ausubel, David Paul, Joseph Novak y Helen Hanesian (1983), Psicología educativa: un punto de vista cognoscitivo, México, Trillas.

Berger, Peter y Thomas Luckmann (2001), La construcción social de la realidad, Buenos Aires, Amorrortu.

Bernasconi, Andrés (2014), La educación superior de Chile: transformación, desarrollo y crisis, Santiago de Chile, Ediciones UC.

Boudon, Raymond (2003), Raisons bonnes raisons, París, PUF.

Bourdieu, Pierre y Jean-Claude Passeron (2009), Los herederos, los estudiantes y la cultura, Buenos Aires, Siglo XXI.

Bruner, Jerome (1972), Hacia una teoría de la instrucción, México, Unión Tipográfica Editorial Hispano Americana.

Brunner, José Joaquín (2013), “De elite a masa a universal: algunos impactos de la masificación", Simposio sobre educación superior de masas, Santiago de Chile, en: http://www.brunner. $\mathrm{cl} / \mathrm{p}=8211$ (consulta: 8 de septiembre de 2020).

Castillo, Jorge y Gustavo Cabezas (2010), “Caracterización de jóvenes primera generación en educación superior. Nuevas trayectorias hacia la equidad educativa", Calidad en la
Educación, núm. 32, pp. 44-76. DOI: https:// doi.org/10.31619/caledu.n32.151

Comisión Nacional de Educación Superior (CNED) (2020), "Matrícula Sistema de Educación Superior”, Santiago de Chile, en: https://www. cned.cl/indices/matricula-sistema-de-educacion-superior (consulta: 8 de septiembre de 2020).

De Juanas Oliva, Ángel y Jesús Beltrán Llera (2014), "Valoraciones de los estudiantes de ciencias de la educación sobre la calidad de la docencia universitaria”, Educación XX1, vol. 17, núm. 1, pp.59-82.DOI: https://doi.org/10.5944/educxx 1.17.1.10705

De Sousa Santos, Boaventura (2005), La universidad en el siglo XXI. Para una reforma democrática y emancipadora de la universidad, Buenos Aires, Miño y Dávila.

Del Valle, Damián, Federico Montero y Sebastián Mauro (comp.) (2017), El derecho a la universidad en perspectiva regional, Buenos Aires, IEC-CONADU/CLACSO.

Freire, Paulo e Ira Shor (2014), Miedo y osadía, Buenos Aires, Siglo XXI.

Giroux, Henry (2016), "La educación superior y las políticas de ruptura", Revista Entramados. Educación y Sociedad, núm, 3, pp. 15-26.

Guerra García, Jorge (2003), "Metacognición: definición y enfoques teóricos que la explican", Revista Electrónica de Psicología Iztacala, vol. 6, núm. 2, en: http://www.revistas.unam. mx/index.php/repi/article/view/21698/20433 (consulta: 8 de septiembre de 2020). 
LARotonda, Christian (2018), Desarrollo histórico de las representaciones sociales acerca del rol de estudiante universitario: un estudio en carreras de las ciencias sociales y las humanidades de universidades no-tradicionales, Tesis de Doctorado, Santiago de Chile, Universidad Academia de Humanismo Cristiano.

Larotonda, Christian (2019), "Continuidades y rupturas en las representaciones sociales de alumnos y profesores acerca del rol de estudiante", Revista Argentina de Educación Superior, vol. 11, núm. 18, pp. 120-135.

Le Boterf, Guy (2001), Ingeniería de las competencias, Barcelona, Ediciones Gestión 2000.

Le Boterf, Guy (2018), Développer et mettre en oeuvre la compétence: Comment investir dans le professionnalisme et les compétences, París, Eyrolles.

LEóntieV, Alexiéi (1985), "Introducción sobre la labor creadora de L.S. Vygotski”, en Obras escogidas I, Madrid, Visor, pp. 417-450.

Linne, Joaquín (2018), "El deseo de ser primera generación universitaria. Ingreso y graduación en jóvenes de sectores populares", Revista Latinoamericana de Educación Inclusiva, vol. 12, núm. 1, pp. 129-147. DOI: https://doi. org/10.4067/S0718-73782018000100129

LONERGAN, Bernard (1992), Insight (A study of human understanding), Toronto, University of Toronto Press.

López Pérez, Lourdes (2018), “El hacer, elemento constitutivo para la construcción de competencias", en Osvaldo Leyva Cordero, Francisco Gangas Contreras, José Tejada Fernández y Abraham Hernández Paz (coords.), La formación por competencias en la educación superior: alcances y limitaciones desde referentes de México, España y Chile, México, Tirant Humanidades, pp. 19-44.

Macchiarola, Viviana (2007), "Curriculum basado en competencias. Sentidos y críticas", Revista Argentina de Enseñanza de la Ingeniería, vol. 8, núm. 14, pp. 38-46.

Morcke, Anne Mette, Tim Dornan y Berit Eika (2013), "Outcome (Competency) Based Education: An exploration of its origins, theoretical basis, and empirical evidence", Advances in Health Sciences Education, núm. 18, pp. 851-863. DOI: https://doi.org/10.1007/s104 59-012-9405-9

Muñoz, Ana Luisa y Valeria Sobrero (2006), "Proyecto Tuning en Chile: análisis de procesos de internacionalización de la educación superior”, Calidad en la Educación, núm. 24, pp. 249-271. DOI: https://doi.org/10.31619/caledu.n24.278

Osorio, Francisco (1999), "El científico social: entre la actitud natural y la actitud fenomenológica”, Cinta de Moebio, núm. 5, pp. 119-128, en: https://www.moebio.uchile.cl/05/osorio011. html (consulta: 8 de septiembre de 2020).
Perrenoud, Philippe (2008), "Construir las competencias, ¿es darle la espalda a los saberes?", Red U. Revista de Docencia Universitaria, vol. 6, núm. 2, número monográfico II: "Formación centrada en competencias", en: https:// revistas.um.es/redu/article/view/35261 (consulta: 8 de septiembre de 2020).

Piaget, Jean (1999), La psicología de la inteligencia, Barcelona, Editorial Crítica.

Ricoeur, Paul (2001), Del texto a la acción, Buenos Aires/México, Fondo de Cultura Económica.

Ricoeur, Paul (2008), Hermenéutica y acción. De la hermenéutica del texto a la hermenéutica de la acción, Buenos Aires, Prometeo.

Ríos, Teresa (2004a), La configuración de sentidos en un grupo de jóvenes liceanos de sectores populares: un acercamiento hermenéutico al mundo juvenil escolar, Tesis de Doctorado, Santiago de Chile, Pontificia Universidad Católica de Chile.

Ríos, Teresa (2004b), "La comprensión del acto de escucha en la educación escolar a partir de la filosofía reflexiva de Paul Ricoeur", $P$ lis, núm. 15, DOI: https://doi.org/10.32735/ S0718-6568/2006-N15-471

Ríos, Teresa (2005), "La hermenéutica reflexiva en la investigación educacional”, Revista Enfoques, Educacionales, vol. 7, núm. 1, pp. 51-66.

Ríos, Teresa (2008), "Los discursos de los y las estudiantes de Talca frente a la docencia universitaria", Cuadernos de Docencia Universitaria, vol. 1, núm. 1, pp. 86-99.

Ríos, Teresa (2013), La hermenéutica reflexiva en la investigación educacional y social, Manizales, Universidad de Manizales/Universidad Academia de Humanismo Cristiano.

Rueda Beltrán, Mario, Norberto Fernández Lamarra, Pablo Bakieva, Margarita García, José González Such, Jesús Miguel Jornet Meliá, Carlos Sancho Álvarez, Alejandro Canales Sánchez, Edith J. Cisneros Cohernour, José Alberto Contreras Eljure, José L. López Osorio, María Cristina Parra Sandoval, Alicia Inciarte, Jesús Alberto Fuenmayor y Ana Julia Bozo de Carmona (2019), "Prácticas y condiciones institucionales para el desarrollo de la docencia en universidades iberoamericanas", Publicaciones, vol. 49, núm. 1, pp. 19-37. DOI: https://doi.org/10.30827/publicaciones.v49i1.9850

Santos Guerra, Miguel Ángel (2003), “Dime cómo evalúas y te diré qué tipo de profesional y de persona eres", Revista Enfoques Educacionales, vol. 5, núm. 1, pp. 69-80.

SснӧN, Donald (1992), La formación de profesionales reflexivos, Barcelona, Paidós.

Schutz, Alfred (2003), Estudios sobre teoría social, Buenos Aires, Amorrortu.

SEn, Amartya (2000), Desarrollo y libertad, Buenos Aires, Planeta. 
Soтo Hernández, Valentina (2016), “Estudiantes de primera generación en Chile: una aproximación cualitativa ala experiencia universitaria”, Revista Complutense de Educación, vol. 27, núm. 3, pp. 1157-1173.

TARDIF, Jaques (2003). "Développer un programme par compétences: de l'intention à la mise en œuvre”, Pédagogie Collégiale, vol. 16, núm. 3, pp. 36-44.

TARDIF, Jaques (2009), “Se o professor universitário fosse uma profissão", en María Isabel da Cunha, Sandra Regina Soares y Marinalba López Ribeira (eds.), Docência universitária: profissionalização e praticas educativas, Feira de Santana (Bahía, Brasil), UEFS Editora, pp. 57-77.

Tejada Fernández, José (2018), "La docencia universitaria en el nuevo contexto enseñanzaaprendizaje por competencias", en Osvaldo Leyva Cordero, Francisco Gangas Contreras, José Tejada Fernández y Abraham Hernández Paz (coords.), La formación por competencias en la educación superior: alcances y limitaciones desde referentes de México, España y Chile, México, Tirant Humanidades, pp. 93-119.

Tenti, Emilio (1993), "Universidad en crisis y el campo intelectual. Entre la autonomía y la excelencia", Revista del Instituto de Investigaciones en Ciencias de la Educación, año 2, núm. 3,pp. 85-89.
VÉLEz Bedoya, Ángel, Luis Delgado Vélez y William Sánchez Torres (2018), “Análisis prospectivo de las competencias genéricas Tuning-Alfa en la ciudad de Medellín al 2032", El Ágora USB, vol. 18, núm. 1, pp. 131-152. DOI: https:// doi.org/10.21500/16578031.3446

Vergara, Jorge (2012), "El debate entre la educación republicana y la neoliberal en Chile", Polisemia, vol. 8, núm. 13, pp. 62-73. DOI: https:// doi.org/10.26620/uniminuto.polisemia. 8.13.2012.62-74

VIdal, Paulina, Jorge Milton Vidal, Jorge Vidal, Camila Morales, Teresa Ríos, Liliana Salazar y Paloma Ramírez (2017), La educación superior chilena como campo de disputa ideológica, Santiago de Chile, Ediciones Universidad Academia de Humanismo Cristiano.

Vygotski, Liev (1985), "Pensamiento y lenguaje”, en Liev Vygotski, Obras escogidas II, Madrid, Visor Distribuciones, pp. 10-286.

Zeichner, Kenneth y Daniel Liston (1993), Formación del profesorado y condiciones sociales de la escolarización, Madrid, Morata. 